\section{Klinischer Score zur Entscheidungshilfe für Typ-I-Interferonopathien}

Sonmez HE, Karaaslan C, de Jesus AA et al. A clinical score to guide in decision making for monogenic type I IFNopathies. Pediatr Res 2000; 87(4): 745-752

Die relativ neue Krankheitsgruppe der autoinflammatorischen Interferonopathien (AIIFN) ist relativ schwierig zu diagnostizieren, da sie ein außerordentlich breites Symptomenspektrum mit teilweise unspezifischen Befunden aufweist. Aus diesem Grund kann ein klinisch orientierter Diagnose-Score hilfreich sein. Während die klassischen Autoinflammationserkrankungen (u. a. Familiäres Mittelmeerfieber, systemische juvenile Arthritis) Interleukin-1 getrieben sind, handelt es sich bei den Interferonopathien um Krankheitsbilder, bei denen es zur Dysregulation der angeborenen IF-Typ-1Homöostase gekommen ist. Unter anderem umfasst diese Gruppe das Aicardi-Goutières Syndrom (AGS), das Krankheitsbild der chronisch atypischen neutrophilen Dermatose mit - subfebrilen - Temperaturen (CANDLE) und der interferonstimulierten Vaskulopathie mit Beginn im Säuglingsalter (SAVI).

In der vorliegenden Arbeit wurde initial eine ausführliche Literaturrecherche durchgeführt mit dem Ziel das Symptom- und Befundspektrum der publizierten Fälle von
Patienten mit Al-IFN zu charakterisieren. In einem 2. Schritt wurden 12 pädiatrische Patienten mit den klinischen Zeichen einer AI-IFN ausgewertet. Dabei hatten alle 12 eine Hautbeteiligung: am häufigsten UIzera, akral lokalisierte Exantheme, Pannikulitiden, Livedo retikularis, Frostbeulenähnliche Veränderungen, digitale Gangrän und Raynaud-Zeichen sowie eine Lipodystrophie. Am zweithäufigsten fand sich eine ZNS-Beteiligung mit Basalganglienverkalkungen, Veränderungen der weißen Substanz, progressiver zerebraler Atrophie und ZNS-Vaskulitis, sowie eine psychomotorische Retardierung. Weiterhin fanden sich Kontrakturen, Arthritiden, interstitielle Lungenerkrankungen und Fibrosen. An Blutbildauffälligkeiten waren Leuko- und Lymphopenien zu sehen.

Aus diesen sowie den Daten der Literaturrecherche wurde ein klinischer Score mit folgenden Kriterien ermittelt:

- Hautmanifestation (noduläres Exanthem, livide Plaques in kältesensitiven akralen Arealen)

- Vaskulopathie (frostbeulenähnliche Effloreszenzen, mikroangiopathische Vakulopathie, akrale Gangrän/Ulzera/ Infarkt)

- Lipodystrophie

- Gelenkmanifestation (Kontraktur, Arthritis)

- Myositis
- ZNS-Manifestation (Basalganglienverkalkung, Leukenzephalopathie, Beteiligung der weißen Substanz, Pleozytose)

- Lungenbeteiligung (interstitielle Veränderungen, Fibrosen, pulmonale Hypertension)

- Leuko-, Lymphopenie

Jedes Kriterium wurde mit 0 bzw 1 gewertet. Dabei zeigte sich, dass - im Gegensatz zu gesunden Kontrollen - alle Patienten mit einer vermuteten AI-IFN einen Score von $\geq 3$ aufwiesen. Auch die Interferonsignatur war signifikant erhöht im Vergleich zu den gesunden Kontrollen. In einer Genanalyse (whole exom sequencing) konnte bei 9 von 12 Patienten mit der vermuteten AI-IFN die Diagnose molekulargenetisch bestätigt werden.

Auch wenn der vorgestellte Score nur vorläufig ist und zukünftig sicherlich noch weiter spezifiziert werden muss, so bietet er dennoch eine Möglichkeit, aus der unklaren und oft vagen Symptomenkonstellation dieser Patienten eine klinische Verdachtsdiagnose zu konstruieren. Das ist die Grundlage für eine gezielte weitergehende Diagnostik (Interferonsignatur, whole-exome sequencing) und die Chance auf die Einleitung einer frühzeitigen Therapie.

Toni Hospach, Zentrum für Pädiatrische Rheumatologie am Klinikum Stuttgart (ZEPRAS), Olgahospital, Stuttgart 